\title{
On the impact of the laser radiation wavelength and the concentration of quantum dots on the luminescence spectra of colloid solution and QD-doped nanocomposites
}

\author{
A.E. Eskova ${ }^{1}$,A.I. Arzhanov ${ }^{1,2}$, K.A. Magaryan $^{1}$, N.A. Koverga ${ }^{1}, K . R$. Karimullin $^{1,2}$, and \\ A.V. Naumov ${ }^{1,2}$
}

${ }^{1}$ Moscow State Pedagogical University, 119435 Moscow, Russia

${ }^{2}$ Institute for Spectroscopy RAS, 108840 Moscow, Troitsk, Russia

\begin{abstract}
The influence of the concentration and the laser excitation wavelength on the width and luminescence spectrum maximum of $\mathrm{CdSe} / \mathrm{CdS} / \mathrm{ZnS}$ quantum dots in the toluene solution and doped on the glass plate was studied. It was shown that the wavelength of the exciting laser does not affect the width and peak center of the luminescence spectrum of the samples under the study. Possible mechanisms of the quantum dots concentration influence on studied parameters are analyzed.
\end{abstract}

The study of the spectral and photophysical properties of semiconductor quantum dots (QDs) is an important task in modern science and materials science [1-6]. To develop some practical applications based on quantum dots, it is necessary to study the temperature dependence of the spectral properties of QDs and composites based on them [7, 8].

We studied CdSe/CdS/ZnS quantum dots (QD Light, Russia) in toluene solution and doped on the glass plate. For the concentration dependence measurements the QD solution in pure toluene with the initial concentration $C_{0}=96 \mu \mathrm{g} / \mu \mathrm{l}$ was used. The concentration of solution was varied by adding a certain amount of toluene to obtain the solutions of 32.0, $19.2,13.7,4.6,2.7,2.0,0.65,0.39,0.28$ and $0.09 \mu \mathrm{g} / \mu \mathrm{l}$. Spectral measurements on solutions were performed using a 1-mm quartz cuvette. Excitation power and wavelength dependence measurements were carried out with the use of QDs on glass plate. This sample was prepared with the blade coating technique that was previously described in [9].

For each sample, the measured luminescence spectra were approximated by the Gauss function, which made it possible to determine the width and the positions of the luminescence peaks from the spectra. The luminescence was excited using a tunable linear dye laser CR-599 (Coherent Radiation). The laser radiation intensity was attenuated by neutral filters. Luminescence spectra were measured on an SDL-1 spectrometer, where a PCO PixelFly CCD camera was used as a detector. All spectra were measured at $\mathrm{T}=300 \mathrm{~K}$.

Fig. 1 shows the luminescence spectra of solutions with different QD concentration. We note that from a certain concentration value the luminescence intensity increase is replaced by a slight decrease (similar dependence was observed in [10]). A decrease in fluorescence intensity with the concentration increase refers to a concentration quenching. With an increase in the concentration of luminescent centers it is rather possible to reach the Förster 
radius between two emitting centers. Besides the change of the luminescence intensity, the luminescence peak center shifts in accordance with the QD concentration in the solution. With the increase in the concentration of QDs in solution, the red-shift is observed, which may be due to the dipole - dipole interaction [11]. Also the concentration dependence of the luminescence spectrum width for the prepared solutions was studied. It was found that the spectra broaden with increasing concentration of QDs.
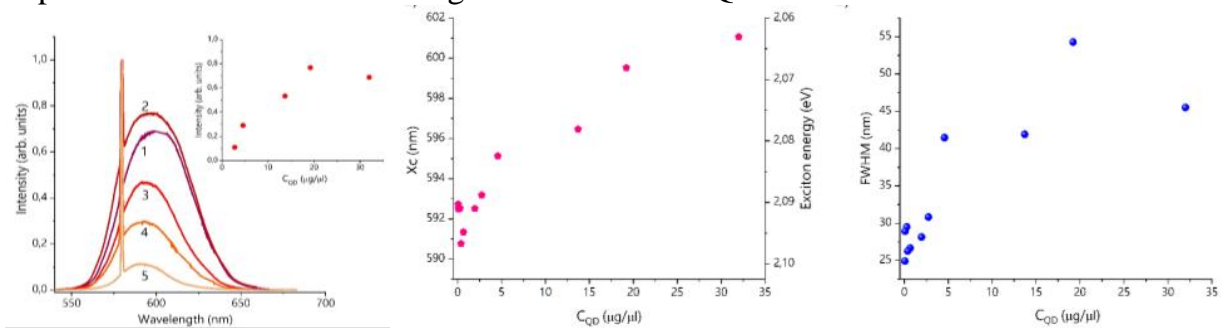

Fig. 1. Luminescence spectra of QD-solutions with intensity normalized to the laser excitation line (narrow line in the left part of the spectrum). The insert shows the concentration dependence of the luminescence spectrum intensity, measured at a maximum. The QD concentration in the samples: 1 32,2 - 19.2, 3 - 13.7, 4 - 4.6, 5 - $2.7 \mu \mathrm{g} / \mu \mathrm{l}$ (on the left). The influence of the QD concentration in toluene on position $X_{\mathrm{c}}$ (in center) and the FWHM (on the right) of the luminescence spectrum.

It follows from the results obtained that the wavelength of the exciting laser has very little effect on the width and position of the luminescence peak center for the sample under the study. However, in [12] the influence of excitation wavelength was observed for quantum rods. Such difference possibly can be explained with QDs structure difference.

The concentration dependences of the peak position (exciton energy) and the width of the luminescence spectrum of solutions with QD in toluene were analyzed. It was shown that the value of the exciton energy, determined by the luminescence spectrum, can vary from 2.065 to $2.1 \mathrm{eV}$ in the concentration range from 0.09 up to $32 \mu \mathrm{g} / \mu \mathrm{l}$. A possible mechanism for shifting the luminescence spectrum of a QD solution with an increase in their concentration is nonradiative energy transfer. An explicit influence of excitation wavelength on the spectral characteristics for the studied sample was not detected.

The studies showed that a significant change in the position and width of the luminescence spectrum depending on the selected concentration of QDs in the sample must be taken into account when conducting temperature measurements and analysis of electronphonon interaction processes.

The reported study was funded by RFBR and Moscow city Government (proj. № 19-32-70005).

\section{References}

1. K.R. Karimullin, A.V. Naumov, J. Lumin. 152, 15 (2014)

2. A.L. Shchukina et al., Phys. Rev. E 92, 032102 (2015)

3. I.Yu. Eremchev et al., J. Phys. Chem. C 120, 22004 (2016)

4. A.I. Arzhanov et al., Bull. Lebedev Phys. Inst. 45, 91 (2018)

5. K.R. Karimullin et al., Bull. Russ. Acad. Sci. Phys. 82, 1620 (2018)

6. K.A. Magarian et al., J. Phys. Conf. Ser. 478, 012007 (2013)

7. K.A. Magaryan et al., Opt. Spectroscopy 126, 41 (2019)

8. K.R. Karimullin et al., J. Phys. Conf. Ser. 951, 012011 (2018)

9. K.R. Karimullin et al., J. Phys. Conf. Ser. 859, 012010 (2017)

10. S.V. Rempel' et al., Phys. Sol. St. 57, 1103 (2015)

11. M. Lunz et al., Phys. Rev. B 81, 205316 (2010)

12. D. Geißler et al., Phys. Chem. Chem. Phys. 19, 12509 (2017) 